Publicación Semestral Pädi Vol. 7 No. 14 (2020) 42-46

\title{
Síntesis de bis(indolil)escuaraínas
}

\author{
Synthesis of bis(indolyl)escuaraines. \\ María del Carmen Aguilar-Gómez ${ }^{\mathrm{a}}$, Mariana Flores-Jarilloa , Alejandro Álvarez-Hernández ${ }^{\mathrm{a}, *}$ \\ a Área Académica de Química, UAEH, Carretera Pachuca-Tulancingo Km. 4.5, Col. Carboneras, 42184 Mineral de la Reforma, Hidalgo, México
}

\begin{abstract}
Resumen
Los colorantes de tejido celular vivo que se utilizan en la microscopia de fluorescencia son sustancias que absorben dos fotones de radiación infrarroja que logra penetración profunda en el tejido sin causar daño a la muestra debido a su baja foto toxicidad. Los derivados de escuaraína son compuestos privilegiados para la preparación de imagen celular porque muestran fluorescencia por la absorción de dos fotones. En este trabajo se describe la síntesis de bis(indolil)escuaraínas simétricas por la reacción de indoles con ácido escuárico en una solución de tolueno-butanol. La reactividad química de las bis(indolil)escuaraínas provocó problemas de reproducibilidad en los rendimientos de reacción y en la purificación de estos productos. Se encontró que el uso de beta ciclodextrina como agente de encapsulamiento de algunas bis(indolil)escuaraínas estabiliza el anillo central y evita reacciones laterales. Sin embargo, el espacio de la cavidad en la beta ciclodextrina parece ser insuficiente para encapsular escuaraínas arilsustituidas en el anillo de 5 miembros del indol como el compuesto 3, en cuya síntesis el uso de la ciclodextrina no presentó ningún beneficio. Las bis(indolil)escuaraínas obtenidas son fluorescentes en solución y en estado sólido. Se determinó que la fluorescencia por absorción de un fotón ocurre por irradiación a $365 \mathrm{~nm}$ y mediante la absorción de dos fotones bajo irradiación con un láser a $710 \mathrm{~nm}$.
\end{abstract}

\section{Palabras Clave:}

Absorción de dos fotones, beta ciclodextrina, escuaraínas, fluorescencia.

\begin{abstract}
Living cell dyes used in fluorescence microscopy are chemical substances capable to absorb two photons of infrared irradiation which allows a deeper tissue penetration without showing cell toxicity. Squaraines display two photon absorption fluorescence, consequently they are privileged compounds for cell image acquiring. In this work the synthesis of symmetrical bis(indolyl)escuaraines from the reaction between squaric acid and several indoles in a mixture of toluene-butanol is described. The chemical reactivity of the squaraine central ring caused problems of isolation and reproduction of chemical yields of reaction products. The use of beta cyclodextrin as encapsulation agent to protect the reactive squaraines $4 \mathrm{a}-\mathrm{d}$ and 5 from side reactions worked well in the condensations reactions of squaric acid with small indoles. The technique allowed the isolation of pure bis(indolyl)squaraines in reproducible yields. However, the limited size of the beta cyclodextrin cavity was unable to host molecules with a larger indole fragment such as 2 . Thus the use of beta-cyclodextrin had no beneficial effect on the synthesis of the bis(indolyl)squaraine 3 .

All squaraines prepared in this work exhibit fluorescence in solution by absorption of one photon of UV light (365 nm) and it was shown that some of these compounds such as $4 \mathrm{c}$ are fluorescent in the solid state too. Furthermore, this compound was shown to absorb two photons of an infrared laser $(710 \mathrm{~nm})$ to emit fluorescence at $575 \mathrm{~nm}$ in solution and $695 \mathrm{~nm}$ in the solid state.
\end{abstract}

Keywords:

Squaraines, fluorescence, two photon absorption, beta cyclodextrin.

\section{Introducción}

Las escuaraínas (Kitson, 2017) son compuestos producidos por la condensación del ácido escuárico 1 con 2 moléculas ricas en electrones tales como anilinas, fenoles, pirroles e indoles, denotados como $\mathrm{X}$ en la Figura 1. Aunque la representación alterna del anillo central de estos compuestos zwitteriónicos (Figura 1, A) es una estructura aromática con doble carga positiva (Figura 1, B), recientemente ha sido rebatida en base a estudios teóricos que indican que la polarización de este anillo en realidad desplaza la carga positiva a los grupos sustituyentes (Divya, 2019)

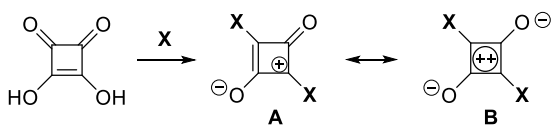

Figura 1. Estructura general de escuaraínas

\footnotetext{
*Autor para la correspondencia: alvarez@uaeh.edu.mx

Correo electrónico: 91 mcag@ @mail.com (María del Carmen Aguilar Gómez), mariana_flores@ uaeh.edu.mx (Mariana Flores Jarillo), alvarez@ uaeh.edu.mx (A. Álvarez-Hernández), 
La principal característica física de estos compuestos es la intensa absorción de luz que ocasiona la emisión de fluorescencia. Estas estructuras privilegiadas tienen varias aplicaciones tecnológicas derivadas de sus propiedades ópticas (Xia y Wang, 2017), por ejemplo: conversión de luz solar (Wickramsinghe, 2019, Yang, 2019), terapia fotodinámica (Sun, 2018, Ramahia, 2002), sondas fluorescentes para la detección de cáncer (Ahn, 2012) y la detección de analitos (Liu, 2019). Algunas estructuras de escuaraínas con su aplicación se muestran en la Figura 2.

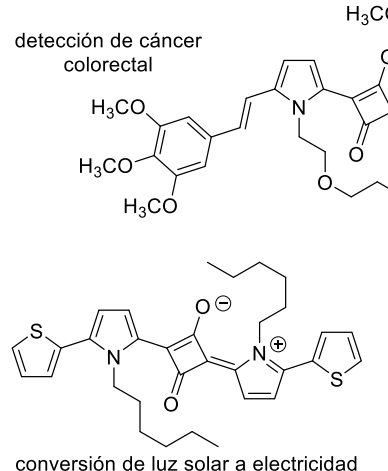

Figura 2. Algunas aplicaciones de las escuaraínas.

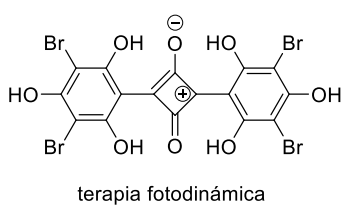

Las escuaraínas han sido motivo de gran interés para preparar sondas fluorescentes para la elaboración de imagen celular con fines biomédicos debido a su capacidad de absorber dos fotones de luz infrarroja. Esta propiedad es especialmente compatible con las células vivas por su baja citotoxicidad y porque a diferencia de la luz visible, el infrarrojo penetra los tejidos y no sufre difracción, lo que genera una imagen con mayor resolución espacial. Además, no hay interferencia por la fluorescencia de biomoléculas tales como proteínas y ácidos nucleicos porque estas moléculas solamente presentan fluorescencia por absorción de un fotón. La fluorescencia es la emisión instantánea de luz de una molécula electrónicamente excitada. Generalmente las moléculas excitadas tienden a perder el exceso de energía por emisión de calor debido a procesos de vibración. Sin embargo, cuando estos movimientos se restringen por una estructura molecular lo suficientemente rígida la energía se pierde mediante la emisión de luz. La excitación electrónica se puede producir por la irradiación con un fotón de alta energía (luz ultravioleta) que promueve a un electrón del estado basal $\left(\mathrm{S}_{0}\right)$ a un estado de mayor energía $\left(\mathrm{S}_{1}\right)$. Alternativamente, algunas moléculas se pueden excitar por la absorción simultánea de dos fotones de baja energía (luz infrarroja). (Figura 3)
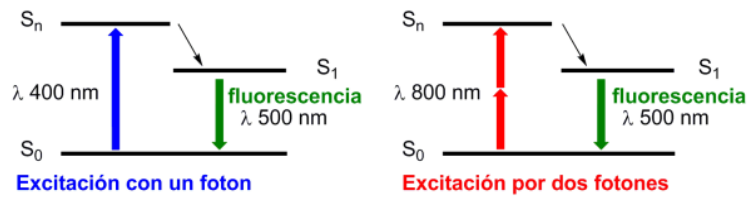

Figura 3. Comparación de la excitación de fluoróforos por la excitación con un fotón (izquierda) y con dos fotones (derecha).

El proceso de absorción de dos fotones fue predicho por María Goeppert-Mayer en 1931 pero sólo se pudo demostrar experimentalmente décadas más tarde con la invención del láser porque este proceso depende del cuadrado de la intensidad de la luz con la que se irradia la muestra. Los estudios enfocados en el diseño de fluoróforos indican algunas características estructurales necesarias (Guo, 2014) para que las moléculas muestren la absorción de dos fotones tales como alta conjugación y polarización electrónica debida a la presencia de un donador- $\pi$ rico en electrones (D) conectado directamente o a través de un fragmento conjugado a un aceptor- $\pi$ (A). La absorción de dos fotones requiere que el estado electrónico excitado esté polarizado respecto al estado electrónico basal (Pawlicki, 2009) y aunque el concepto de diseño es simple, en la práctica la eficiencia del fluoróforo depende de dos parámetros contrapuestos: el rendimiento de fluorescencia (porcentaje de fotones emitidos en relación a los absorbidos) que se abate por el exceso de polarización electrónica y la eficiencia de absorción de dos fotones que se incrementa a medida que la polarización en el estado electrónico excitado aumenta y que repercute en la intensidad de la luz emitida. En este sentido, las escuaraínas son moléculas privilegiadas porque presentan intensa fluorescencia por la absorción de dos fotones de radiación visible o infrarroja. A pesar del excelente carácter donador del indol los ejemplos de bis(indolil)escuaraínas reportados en la literatura aún son escasos (Sun, 2015; Lynch, 2018) en comparación a otros tipos de escuaraínas. (Beverina, 2009). Por lo tanto se decidió efectuar la síntesis de bis(indolil)escuaraínas como materiales fluorescentes con posible aplicación optoelectrónica.

\section{Experimental}

\subsection{Síntesis de indolil escuaraínas}

Preparación del compuesto 3. En un matraz adaptado con una trampa de Dean-Stark con refrigerante para la destilación azeotrópica de agua, se agregó $23 \mathrm{mg}$ (0.2 mmol, 1 equivalente) del ácido escuárico 1 y $171 \mathrm{mg}(0.4 \mathrm{mmol}, 2$ equivalentes) del indol 2 en $15 \mathrm{ml}$ de una mezcla $1: 1 \mathrm{v} / \mathrm{v}$ de $\mathrm{n}$ butanol y tolueno y se calentó a reflujo durante 16 horas según se indica en la Figura 4. La mezcla de reacción inicialmente incolora se torna azul. El disolvente se evaporó parcialmente por destilación a presión ambiental y el sólido depositado en las paredes del matraz de reacción se recuperó como un polvo verde obscuro con brillo metálico. Los datos espectroscópicos se muestran a continuación: IR $\left(\mathrm{KBr}, \mathrm{cm}^{-1}\right) 2923$ (vCsp3- H), 2853 (vCsp2-H), 2228 ( $v$ Csp-N), 2212 (vCsp-Csp), $1744(v \mathrm{C}=\mathrm{N})$, $1624(\mathrm{vC}=\mathrm{O}), 1600$ y 1460 ( $\mathrm{VC}=\mathrm{C}), 1225$ y 1209 (vC-COC), 843 $(\tau \mathrm{C}-\mathrm{H}) ; \mathrm{RMN}^{1} \mathrm{H}\left(400 \mathrm{MHz}, \mathrm{CDCl}_{3}\right): \delta 8.94(\mathrm{~d}, \mathrm{~J}=8.69,1 \mathrm{H}), 7.81$ $(\mathrm{d}, \mathrm{J}=8.25,2 \mathrm{H}), 7.66(\mathrm{~m}, 4 \mathrm{H}), 7.55-7.51,(\mathrm{~m}, 4 \mathrm{H}), 4.05(\mathrm{t}, \mathrm{J}=7.68$, $2 \mathrm{H}), 1.72(\mathrm{q}, \mathrm{J}=6.83,2 \mathrm{H}), 1.18(\mathrm{~m}, 6 \mathrm{H}), 0.83(\mathrm{t}, \mathrm{J}=6.90,3 \mathrm{H})$; $\mathrm{RMN}{ }^{13} \mathrm{C}\left(100 \mathrm{MHz}, \mathrm{CDCl}_{3}\right): \delta 194.0(\mathrm{C}=\mathrm{O}), 148.5,137.6,134.6$, $132.1,132.1,131.6,131.3,128.0,127.3,126.4,118.8,118.5$, $118.3,114.1,113.9,113.4,111.6,94.2,88.3,45.4,31.0,29.5$, $26.4,22.4,13.9$.
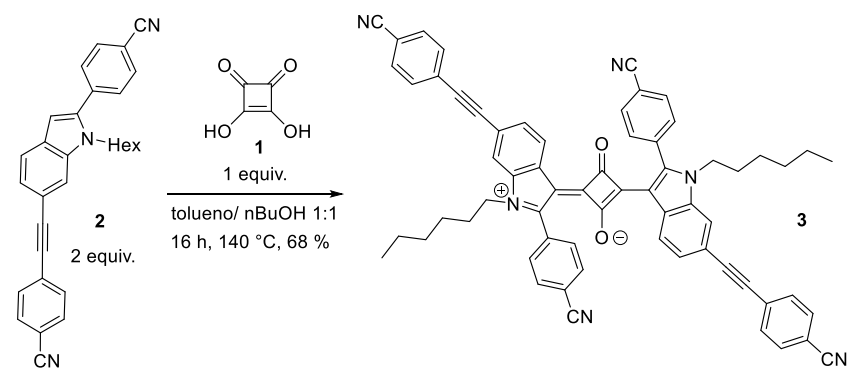

Figura 4. Síntesis de la bis(indolil)escuaraína 3 
La preparación de los compuestos 4a-d y 5 se llevó a cabo usando la misma metodología a partir del ácido escuárico y dos equivalentes del indol correspondiente. (Figura 5)

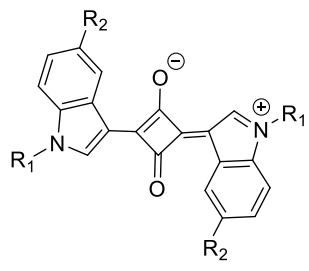

4a. $\mathrm{R}_{1}=$ hexilo $\mathrm{R}_{2}=\mathrm{Br}$

4b. $\mathrm{R}_{1}=$ fenilo $\mathrm{R}_{2}=\mathrm{Br}$

4c. $R_{1}=$ bencilo $R_{2}=B r$

4d. $\mathrm{R}_{1}=\mathrm{H} \quad \mathrm{R}_{2}=\mathrm{H}$

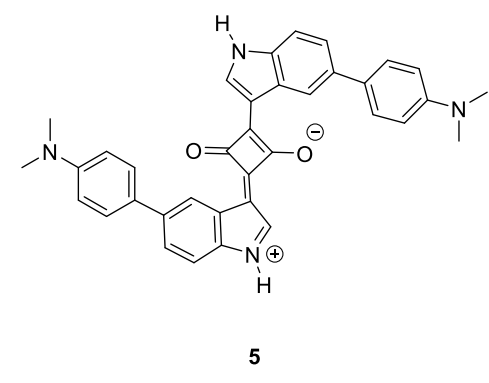

Figura 5. Estructura de las escuaraínas 4 y 5
El compuesto 4a es un sólido de color verde metálico, mientras en solución posee un color rosa intenso (Figura 5) RMN de ${ }^{1} \mathrm{H}\left(400 \mathrm{MHz}, \mathrm{CDCl}_{3}\right) \delta 9.00(\mathrm{~d}, \mathrm{~J}=24.9 \mathrm{~Hz}, 2 \mathrm{H}), 8.61(\mathrm{~d}, \mathrm{~J}=$ $27.1 \mathrm{~Hz}, 2 \mathrm{H}), 7.48(\mathrm{~s}, 1 \mathrm{H}), 7.27(\mathrm{~d}, \mathrm{~J}=9.4 \mathrm{~Hz}, 1 \mathrm{H}), 4.24(\mathrm{t}, \mathrm{J}=$ $7.2 \mathrm{~Hz}, 2 \mathrm{H}), 1.99-1.94(\mathrm{~m}, 1 \mathrm{H}), 1.42-1.29(\mathrm{~m}, 7 \mathrm{H}), 0.91(\mathrm{t}, \mathrm{J}=$ $6.9 \mathrm{~Hz}, 4 \mathrm{H})$.

El compuesto 5 se obtuvo como un sólido azul oscuro. RMN de ${ }^{1} \mathrm{H}\left(400 \mathrm{MHz}, \mathrm{CDCl}_{3}\right) \delta 8.96(\mathrm{~d}, \mathrm{~J}=8.6 \mathrm{~Hz}, 1 \mathrm{H}), 7.84(\mathrm{~d}, \mathrm{~J}=$ $8.1 \mathrm{~Hz}, 1 \mathrm{H}), 7.73-7.64(\mathrm{~m}, 5 \mathrm{H}), 7.58-7.53(\mathrm{~m}, 3 \mathrm{H}), 4.70(\mathrm{t}, \mathrm{J}=$ $6.6 \mathrm{~Hz}, 2 \mathrm{H}), 1.81(\mathrm{dt}, \mathrm{J}=20.4,6.3 \mathrm{~Hz}, 3 \mathrm{H}), 1.49(\mathrm{dt}, \mathrm{J}=14.9,7.4$ $\mathrm{Hz}, 4 \mathrm{H}), 1.00$ (t, J = 7.4 Hz, 4H).

\subsection{Emisión en solución y estado sólido.}

Se preparó una disolución del compuesto con una concentración de $1 \times 10^{-5} \mathrm{M}$ en THF. La calibración del espectrofluorímetro se realizó con soluciones estándar de rodamina $\mathrm{G}$. La medición correspondiente al compuesto en estado sólido se realizó con $10 \mathrm{mg}$ de muestra.

\section{Resultados}

El fragmento donador de indol en la escuaraína 3 se seleccionó de una serie de indoles con alto rendimiento cuántico de fluorescencia previamente reportados (Flores-Jarillo, 2016). La escuaraína 3 en solución en cloroformo tiene un intenso color azul brillante mientras que en estado sólido tiene un color verde metálico. Este comportamiento ha sido documentado y se adjudica a la formación de plasmones por interacción de la luz en la superficie cristalina de las escuaraínas. (Cacciola, 2014). Algunos de los compuestos análogos presentaron un comportamiento similar. Por ejemplo, se ilustra el color de la escuaraína 4a en estado sólido y en solución en diclorometano en la Figura 6.
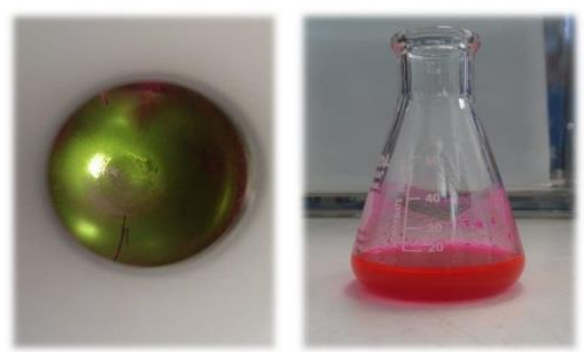

Figura 6. La escuaraína 4a en estado sólido y en solución en $\mathbf{C H}_{2} \mathbf{C l}_{2}$.
La purificación de las escuaraínas presentó problemas de reproducibilidad. Cuando los compuestos se aislaron por simple evaporación del disolvente a presión reducida y precipitación, los espectros de $\mathrm{RMN}$ de ${ }^{1} \mathrm{H}$ de los compuestos crudos generalmente muestran señales múltiples en la región de 2-4 ppm que no corresponden a la estructura de los compuestos deseados. En estos casos, los intentos de purificación por cromatografía en columna (fase normal o inversa) resultaron infructuosos. Los resultados indican que estos productos inseparables probablemente resultan de la adición de butanol a los carbonos de carbonilo del anillo central de la escuaraína. La adición de nucleófilos al anillo de las escuaraínas está ampliamente documentado (Liu, 2019; Ma, 2019, Ros-Lis 2002). Las reacciones laterales indeseadas son notorias en la formación de bis(indolil)escuaraínas. (Lynch, 2018) y por esta razón se probaron diferentes técnicas de aislamiento que se describen a continuación.

Precipitación y cristalización. Se logró precipitar las escuaraínas con éter etílico pero no se logró cristalizarlas por difusión líquido-líquido. Con el uso de otros disolventes como THF y hexano las escuaraínas tienden a descomponerse lentamente. De la misma manera, se observa que en solución de THF se descomponen gradualmente por exposición a la luz y al aire. Sin embargo, las escuaraínas son solubles y estables en solución de cloroformo y diclorometano. La disolución en acetona caliente y cristalización por enfriamiento produce material sólido de pureza variable.

Lavados y filtración. Se hicieron lavados de los productos precipitados con diversos disolventes tales como hexano caliente; etanol, ácido acético y agua en los cuales las escuaraínas tienen una solubilidad limitada a temperatura ambiente y no resultaron efectivos.

Cromatografía. Se intentó cromatografía flash en columna con gel de sílice en fase normal o en fase inversa $\left(\mathrm{C}_{18}\right)$. En ninguno de los casos se pudo obtener material puro, los espectros de RMN de ${ }^{1} \mathrm{H}$ muestran señales en la región de 2.5 ppm que no corresponden a la estructura deseada y sugieren que estas señales no se deben a impurezas presentes en los compuestos sino a grupos unidos covalentemente a la escuaraína. La cromatografía preparativa en placa fina también arrojó resultados insatisfactorios.

Estos resultados apuntan a la formación de productos indeseados por reacciones del anillo de escuaraína durante la síntesis y por tanto se intentó llevar a cabo la formación de estas moléculas por mecano síntesis en la ausencia de disolvente. En tal evento, dos equivalentes de indol 4a-e y uno de ácido escuárico se molieron manualmente en un mortero a temperatura ambiente o a $80^{\circ} \mathrm{C}$. Invariablemente solo se obtuvo el producto de mono condensación según lo revelan los espectros de RMN de ${ }^{1} \mathrm{H}$. Se obtuvo el mismo resultado al efectuar la reacción en etanol a reflujo.

Encapsulación. Se decidió intentar encapsular el núcleo de ácido escuárico con beta ciclodextrina durante la reacción de síntesis para limitar las reacciones laterales del anillo de 4 miembros y facilitar la purificación de los productos. Por ejemplo, el uso de rotaxanos ha demostrado incrementar notablemente la estabilidad de este tipo de compuestos. (Podgosrki, 2012) Aunque las ciclodextrinas son solubles en agua su cavidad interna no es polar y permite formar compuestos tipo anfitrión-huésped con moléculas hidrófobas mediante interacciones no covalentes. La preparación de los compuestos $\mathbf{4 d}$ y $\mathbf{5}$ se seleccionó como modelo 
para explorar la utilidad de la técnica. Se pesó 1 equivalente del ácido escuárico y 1 equivalente de beta ciclodextrina en una mezcla de tolueno y butanol como disolvente y la mezcla de reacción se dejó a reflujo durante 1 hora, luego se agregó 2 equivalentes del indol correspondiente y se continuó con el reflujo durante 6 horas. Finalmente, se redujo el disolvente a presión reducida y se agregó éter etílico frío para precipitar el producto correspondiente y luego se efectuó un lavado con agua. Con satisfacción se observó que ambas escuaraínas 4d y 5 se obtuvieron totalmente puras, se muestra como ejemplo el espectro de $\mathrm{RMN}{ }^{1} \mathrm{H}$ del compuesto 4d. (Figura 7) Sin embargo, el método no fue aplicable a la síntesis de la escuaraína 3 probablemente debido a que la cavidad de la beta ciclo dextrina no es lo suficientemente grande para hospedar a un indol y a una escuaraína de mayor tamaño.

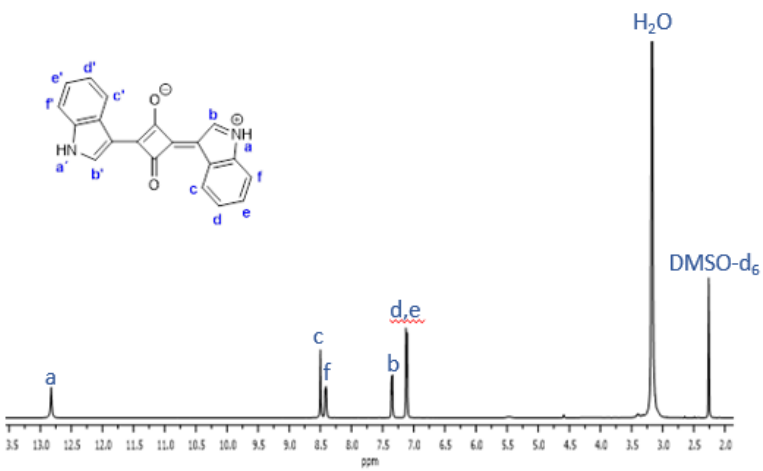

Figura 7. Espectro de RMN de ${ }^{1} \mathrm{H}$ (400 MHz, DMSO-d6) del compuesto 4d obtenido por encapsulación con beta ciclodextrina

Después de la síntesis se pasó al estudio de propiedades ópticas. Los espectros de absorción del indol 2 y la bis(indolil)escuaraína 3 se muestran en la Figura 8. Se observó que el indol 2 tiene una intensa banda de absorción a $364 \mathrm{~nm}$ mientras que el compuesto 3 en solución en cloroformo muestra dos intensas bandas de absorción, una en la región del ultravioleta $(328 \mathrm{~nm})$ y otra en la región del visible $(619 \mathrm{~nm})$. Todas las escuaraínas que se prepararon absorben luz ultravioleta $(365 \mathrm{~nm})$ y emiten fluorescencia azul (Figura 9).

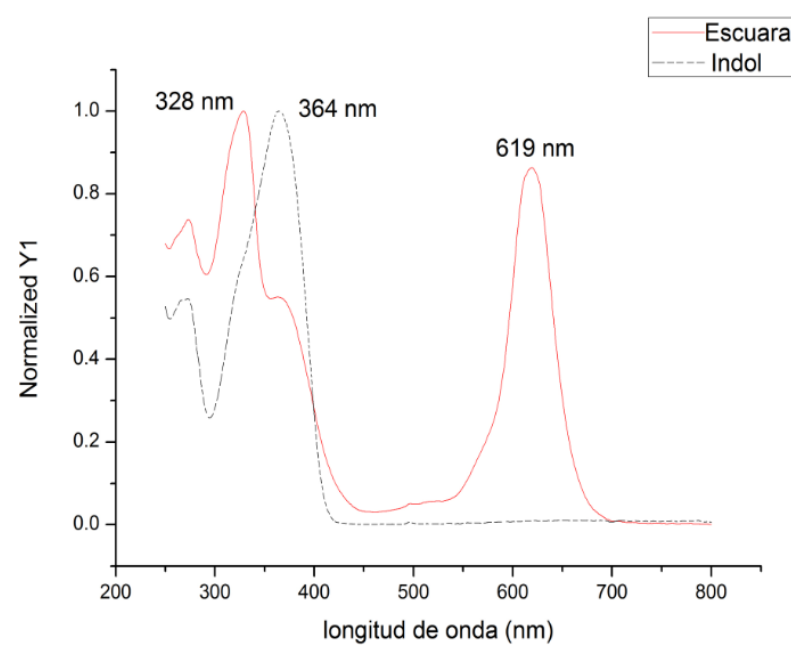

Figura 8. Espectro de absorción del indol 2 y la escuaraína 3 en $\mathrm{CHCl}_{3}$.

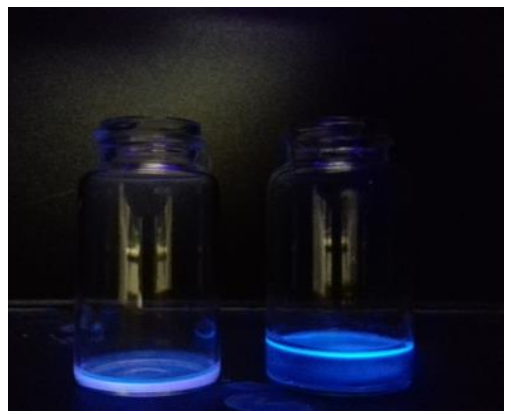

Figura 9. Fluorescencia de los compuestos 3 (derecha) y 4a (izquierda) por irradiación a $365 \mathrm{~nm}$

A continuación, se determinó de manera preliminar si los compuestos realmente emiten fluorescencia por la absorción de dos fotones. Para este estudio se escogió la bis(indolil)escuaraína 4c que es un sólido estable de color púrpura y que mantiene el mismo color en solución en THF. Este compuesto fue irradiado con un láser a diferentes longitudes de onda desde 680 hasta 810 $\mathrm{nm}$. Se observa que el compuesto $\mathbf{4 c}$ emite fluorescencia a una longitud de onda de $575 \mathrm{~nm}$ alcanzando la mayor intensidad de emisión por irradiación a $710 \mathrm{~nm}$ que equivale a la excitación con un fotón de luz ultravioleta a $355 \mathrm{~nm}$ o dos fotones de $710 \mathrm{~nm}$ (luz roja visible). Esta observación concuerda con la intensa banda de absorción en UV que muestra esta serie de compuestos entre 328$365 \mathrm{~nm}$. (Figura 10)

El compuesto $\mathbf{4 c}$ es fluorescente, incluso en estado sólido y tiene un rendimiento cuántico de $25.4 \%$. Cuando se irradió con un láser a $710 \mathrm{~nm}$ emitió fluorescencia a una longitud de onda de $695 \mathrm{~nm}$, en la región límite del espectro visible. En la mayoría de los casos es común que los compuestos en estado sólido emitan fluorescencia a mayor longitud de onda respecto a la emisión en el estado líquido. El desplazamiento en la longitud de emisión se debe a los cambios en las propiedades electrónicas que ocurren por el apilamiento en las moléculas del fluoróforo en estado sólido y su comportamiento como agregado molecular en lugar de moléculas aisladas en solución.

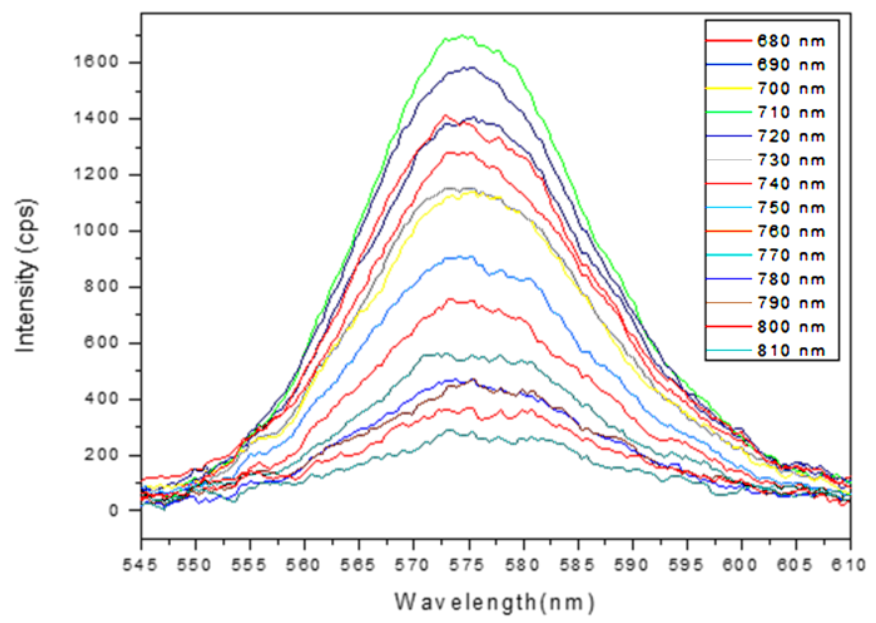

Figura 10. Espectro de emisión de $4 \mathbf{c}$ en solución en THF por irradiación con un láser a diferentes longitudes de onda en el rango 680-810 nm. 


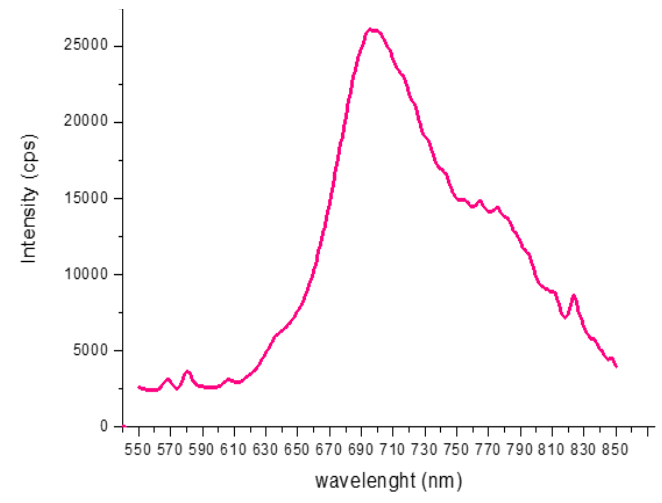

Figura 11. Espectro de emisión de 4c en estado sólido

\section{Conclusión}

Se preparó una serie de bis(indolil)escuaraínas fluorescentes mediante la reacción de indoles con ácido escuárico. Aunque la técnica de preparación es sencilla, la reactividad química de el anillo central de las escuaraínas causa problemas de reproducibilidad en los rendimientos de reacción y la purificación de los productos. El encapsulamiento de los productos de reacción in situ con beta ciclo dextrina permitió aislamiento, purificación y reproducibilidad satisfactoria en el caso de los compuestos 4a-d y $\mathbf{5}$. Sin embargo, posiblemente debido al mayor tamaño molecular de la escuaraína 3 la cavidad de la beta ciclo dextrina es insuficiente para hospedar y proteger a este compuesto de reacciones laterales.

Las escuaraínas sintetizadas presentan fluorescencia por absorción de un fotón de luz UV (365 nm). Además estos compuestos presentan una intensa banda de absorción en el visible, alrededor de $575 \mathrm{~nm}$ para los compuestos 4a-d y 5 y de $619 \mathrm{~nm}$ para el compuesto 3. Experimentalmente se determinó que el compuesto modelo $\mathbf{4} \mathbf{c}$ presenta fluorescencia por absorción de dos fotones de longitud de onda de $710 \mathrm{~nm}$ y tiene potencial como posible marcador de tejidos en la microscopia de fluorescencia.

\section{Agradecimientos}

Este trabajo ha sido realizado gracias al apoyo del Consejo
Nacional de Ciencia y Tecnología (CONACYT) por una beca de maestría a MCAG y apoyo al proyecto CB-2014-221360. Se agradece la asistencia técnica del Dr. Gabriel Ramos Ortiz del Centro en Investigaciones Ópticas para los estudios ópticos de estos compuestos.

\section{Referencias}

Cacciola, A.; Di Stefano, O.; Stassi, R.; Saija, R.; Savasta, S. (2014) Ultrastrong Coupling of Plasmons and Excitons in a Nanoshell. ACS Nano, 8(11), 11483-11492.

Divya, V.V.; Suresh, C. H. (2019) Electronic Structure of Bis(4dimethylaminophenyl)squaraine. Chemistry Select, 4(12), 3387-3394.

Kitson, S. L. (2017) Squaryl Molecular Metaphors Application to Rational Drug Design and Imaging Agents. Journal of Diagnostic Imaging in Therapy, 4(1), 35-75.

Liu, T.; Yang, L.; Zhang, J.; Liu, K.; Ding, L.; Peng, H.; Belfield, K. D.; Fang, Y. (2019) Squaraine-hydrazine adducts for fast and colorimetric detection of aldehydes in aqueous media. Sensors \& Actuators: B. Chemical, 292, 88-93.

Luca Beverina, L.; Salice, P. (2010) Squaraine Compounds: Tailored Design and Synthesis towards a Variety of Material Science Applications. Eur. J. Org. Chem. 1207-1225.

Lynch, D. E.; Hamilton, D. G. (2018) Pyrrol-3-yl squaraines (including indol-3-yl squaraines). J. Heterocyclic Chem., 55, 1249.

Ma, J.; Pang, Z.; Yang, L.; Lu, Z.; Huang, Y.; (2019) The effect of substituents on the stability of the central four-member ring of unsymmetrical squaraine derivatives. Journal of Photochemistry and Photobiology A: Chemistry 376, 160-165.

Podgorski, K.; Terpetschnig, E.; Klochko, O. P.; Obukhova, O. M.; Haas, K. (2012) Ultra-Bright and Stable Red and Near-Infrared Squaraine Fluorophores for In Vivo Two-Photon Imaging. PloS One, 7(12), e51980.

Ramaiah , D.; Eckert , I.; Arun , K. T.; Weidenfeller, L.; Epe, B. (2002) Squaraine Dyes for Photodynamic Therapy: Study of Their Cytotoxicity and Genotoxicity in Bacteria and Mammalian Cells. Photochemistry and Photobiology, 76(6), 672-677.

Ros-Lis, J. V.; Martínez-Máñez, R., Soto, J. (2002) A selective chromogenic reagent for cyanide determination. Chem. Commun., 2248-2249.

Sun, C-L.; Liao, Q.; Li, T.; Li, J.; Jiang, J-Q.; Xu, Z-Z.; Wang, X-D.; Shen, R.; Bai,D-C.; Wang, Q.; Zhang, S-X.; Fube H-B.; Zhang H-L. (2015) Rational design of small indolic squaraine dyes with large two-photon absorption cross section. Chem. Sci., 6, 761-769.

Sun, P.; Wu, Q.; Sun, X.; Miao, H.; Deng, W.; Zhang, W.; Fan, Q.; Huangc, W. (2018) J-Aggregate squaraine nanoparticles with bright NIR-II fluorescence for imaging guided photothermal therapy. Chem. Commun., 54, 13395-13398.

Wickramsinghe, P.; Deokar, P.; Djurovich, P. I.; Haiges, R.; Thompson, M. E. (2019) Symmetric pyrrolic squaraines and their application to organic photovoltaics. Journal of Photochemistry and Photobiology A: Chemistry, 374, 16-21

Xia, G.; Wang, H. (2017) Squaraine dyes: The hierarchical synthesis and application in optical detection. Journal of Photochemistry and Photobiology C: Photochemistry Reviews, 31, 84-113. 